\title{
Anomalous Line Shifts on the SOHO/CDS NIS Detector
}

\author{
S. V. H. Haugan \\ ESA Space Science Department, Goddard Space Flight Center, Mail \\ Code 682.3, Bldg.26 Room G-1, Greenbelt, Maryland 20771, USA
}

\begin{abstract}
Observations with the SOHO/CDS NIS detector prior to the recovery of SOHO showed strong correlations between line shifts and local intensity gradients along the slit, probably caused by an elliptical, tilted point spread function. This must be taken into account when interpreting NIS observations with strong intensity gradients. An initial analysis of post-recovery data does not display the same pattern, indicating that this particular problem was eliminated by changes in the optics during SOHO's "vacation".
\end{abstract}

\section{Introduction}

Haugan (1999) pointed out that data from the SOHO/CDS NIS detector (Harrison et al. 1995) had a tendency to show line shifts wherever the intensity falls or rises steeply, with opposite line shifts on each side of local intensity maxima/minima. A possible solar origin related to rotational flows in helical flux ropes would have predicted opposite patterns for the North and South hemispheres, given a hemispherical preference for handedness in flux ropes (Kumar 1996). However, the pattern was identical in the North and South hemispheres, with blueshifts/redshifts on the North/South side of local intensity maxima, respectively. In the absence of any plausible Solar origin for the observed pattern, it was attributed to a tilted elliptical effective point spread function.

A statistical correction of the observed anomaly was later given in an IDL routine (cds_corr_gradient, part of the standard CDS analysis software). Although a restoration of the true line positions cannot be guaranteed, the correction may be used to gauge the validity of a given result.

Since the optical characteristics of SOHO/CDS changed markedly after the SOHO recovery, a change in this behaviour was expected.

\section{Observations and data analysis}

Haugan (1999) used the synoptic observations performed by CDS every day to explore the nature of the correlations. Results for He I 584 from data prior to the SOHO recovery is shown on the bottom half of Figure 1. It shows the average line intensity (top panel of $7 \times 3$ plots), position (middle panel), and width (lower panel) as a function of the normalized local intensity gradient, calculated relative to points further down the slit (left side of each panel), further up (right 
side), or a central average (middle). The three strips in each panel refer to observations from the northern hemisphere, sun center, and southern hemisphere (top to bottom). For details about the observations, data selection, reduction and analysis, refer to Haugan (1999).

A similar data analysis was performed with more recent data, shown in the top half of Figure 1 for He I 584. Figure 2 shows the same plots as Figure 2 for the $\mathrm{O} v 629$ line.

\section{Discussion}

For He I 584 and O v 629 data taken before the SOHO recovery, there is a large asymmetry in the line position as a function of the normalized local intensity gradient. Haugan (1999) demonstrated that an (almost) elliptical, tilted PSF can reproduce the observations in a qualitative way, and argues that care must be taken when interpreting line shifts in data with moderate to large intensity gradients.

There is also a pronounced effect imposed on the line widths. The line widths measured with the NIS detector are largely instrumental in nature, but if line widths are used, observers should be aware of this anomaly.

Comparing pre- and post-recovery data, we find a very large difference. Apparently, the induced line shifts are much smaller after the recovery, and the effect on line widths appears to have been reversed. The induced line shifts in post-recovery data appear to be so small that a polynomial correction is not warranted.

The Mg IX 368 line, which falls in a differen wavelength band than the other two lines, was also analyzed by Haugan (1999), but only a very small signal was found for this line. This appears to be the case after the recovery as well.

Figure 3 shows the results of applying the recommended correction to the measured line positions in the pre-recovery data set, for He I (upper panel) and $\mathrm{O} \vee$ (lower panel). The asymmetries have been reduced by almost an order of magnitude by a simple linear correction, and no further refinement appears necessary.

Acknowledgments. The author would like to thank all the members of the international CDS team for their dedication in developing and operating the instrument and the associated software. SOHO is a project of international cooperation between ESA and NASA.

\section{References}

Harrison, R. A. et al.: 1995, Solar Phys. 162, 233.

Haugan, S. V. H.: 1999, Solar Physics, 185, 275.

Kumar, A.: 1996, Bull. American Astron. Soc. 188, 8601. 


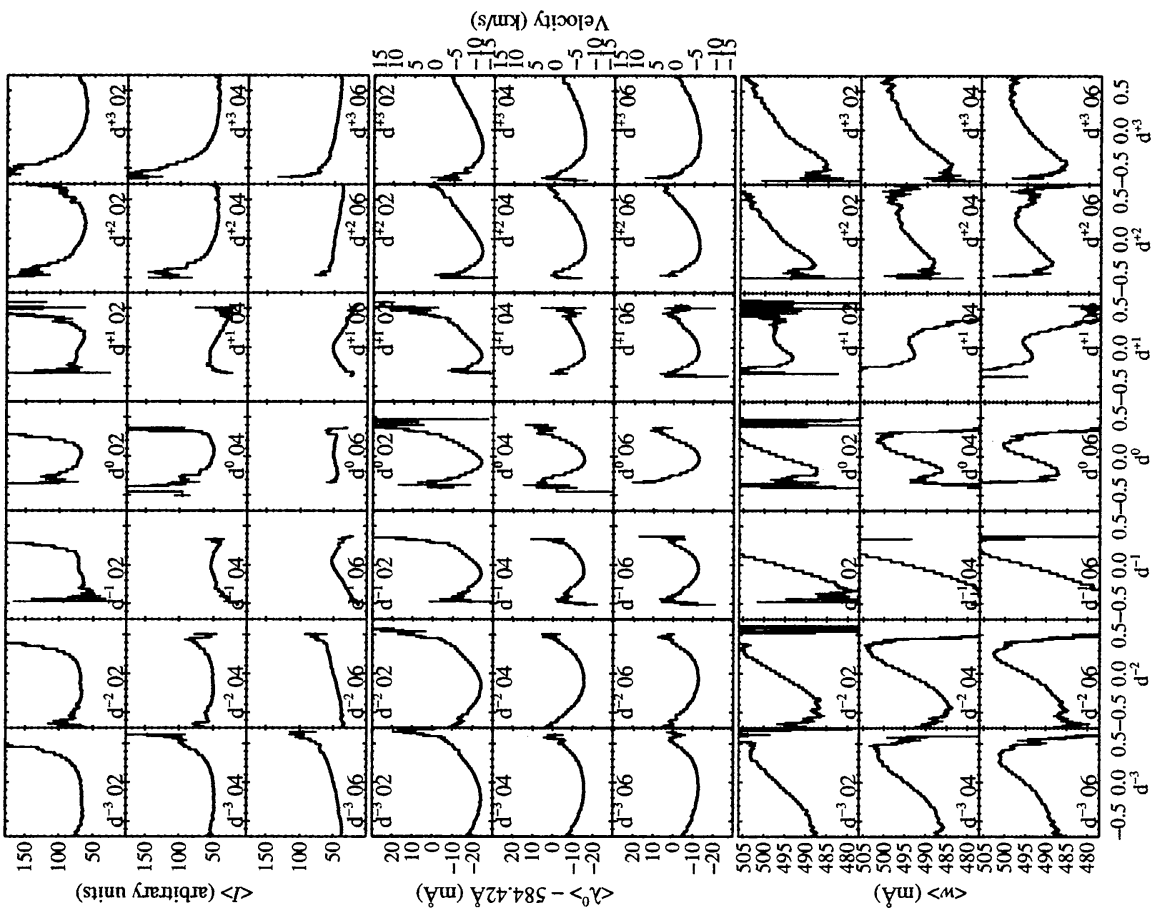

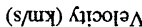

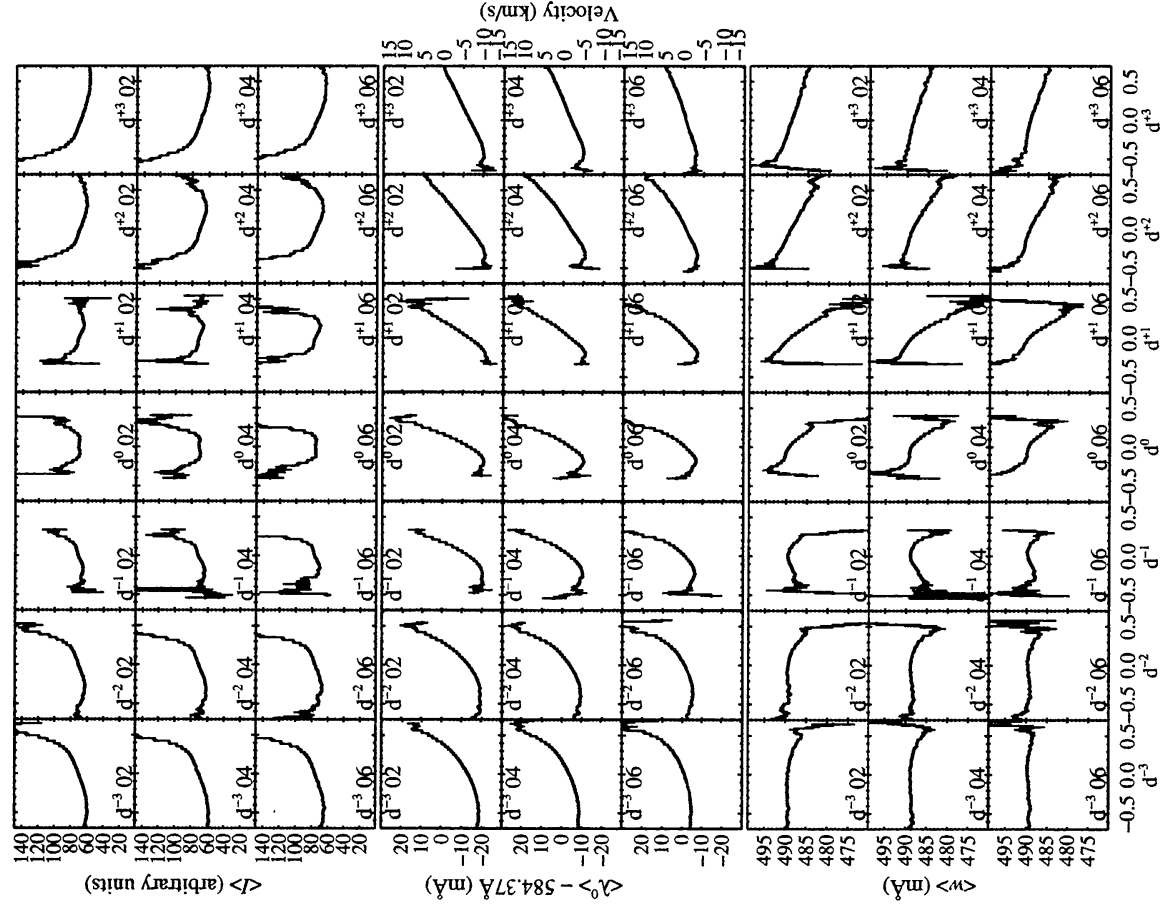

Figure 1. He I 584 data taken before (bottom) and after (top) the SOHO recovery. See Section 2 for an explanation of the plots. 

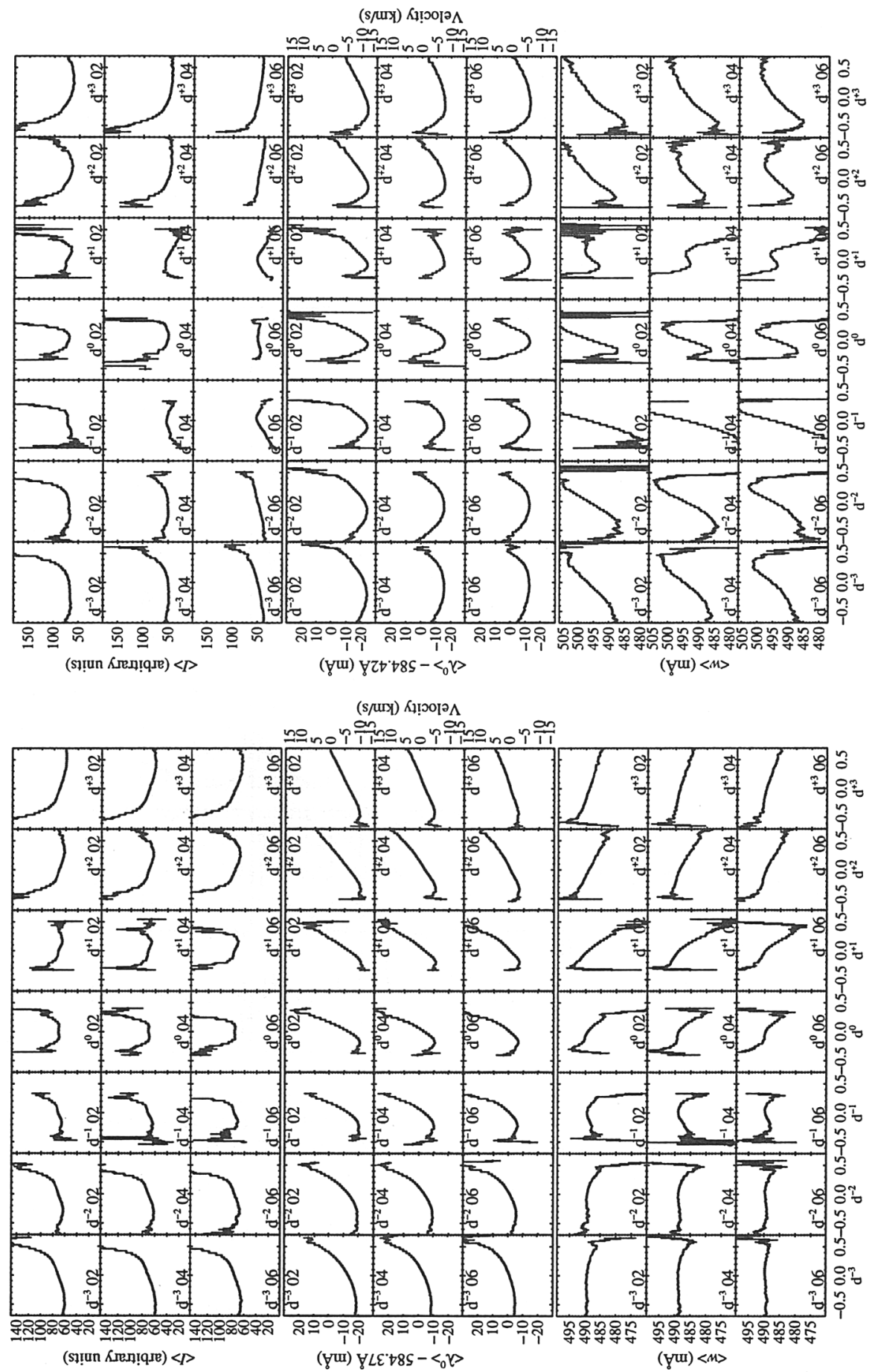

Figure 2. $\quad \mathrm{O} \vee 629$ data taken before (bottom) and after (top) the SOHO recovery. See Section 2 for an explanation of the plots. 

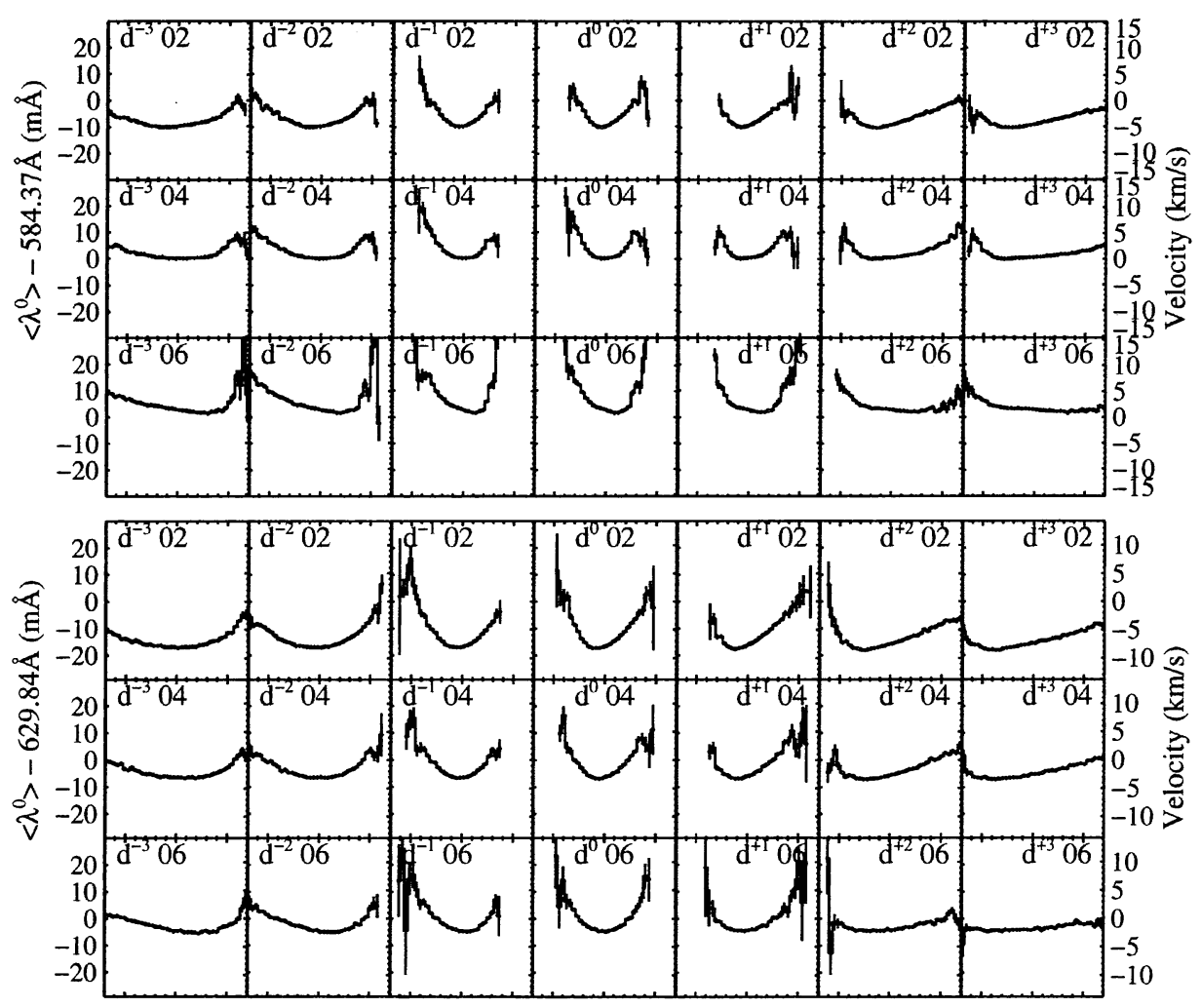

Figure 3. Line position plots for pre-recovery data, after applying a simple linear correction (cds_corr_gradient). He I (upper panel) and O v (lower panel). 